\title{
Sarcopenia and chemotherapy dosing in obese patients
}

\section{Alessandro Laviano, Serena Rianda and Filippo Rossi Fanelli}

We read with interest the Review by Lyman and Sparreboom (Chemotherapy dosing in overweight and obese patients with cancer. Nat. Rev. Clin. Oncol. 10, 451-459; $2013)^{1}$ because obesity is an area of interest for oncologists owing to its association with increased risk of many cancer types. Compared with nonobese individuals, obese patients with cancer face a number of specific challenges related to the diagnosis and treatment of the disease. One such challenge is the optimal dosing of chemotherapy; uncertainties remain regarding toxicity and overdosing of chemotherapy in these patients. ${ }^{1}$ In their article, Lyman and Sparreboom ${ }^{1}$ discussed the evidence supporting the ASCO recommendations for chemotherapy dosing in obese and overweight patients with cancer. ${ }^{2}$ The authors acknowledged that chemotherapy dosing in adult patients with cancer is generally based on body surface area (BSA) and that obese patients receiving full chemotherapy doses do not experience more adverse events than healthy weight individuals. ${ }^{1}$ However, we feel that the recommendations by ASCO to use actual body weight for chemotherapy dosing of overweight and obese cancer patients might not completely acknowledge all the available evidence.

One of the major confounding factors in the clinical approach to treating obese patients across different settings-including medical oncology-is the discrepancy between the definition of obesity and how it is diagnosed in daily practice. The hallmark of obesity is the pathological increase of adiposity, yet obesity is generally diagnosed using BMI, which is calculated using only weight and height. ${ }^{3}$ Thus, the complexity of body composition changes is overlooked and lost. It should be noted that BMI was originally developed for epidemiological studies and its clinical relevance must be cautiously assessed when used for the assessment of individual patients. ${ }^{3}$ Further complicating the clinical scenario in many obese patients is the observation that increased fat mass is associated with sarcopenia, the loss of muscle mass. ${ }^{4}$ The pathogenesis of sarcopenia is complex and related to the ageing process and to diseaseassociated muscle wasting. ${ }^{5}$ In the clinical setting, sarcopenia is prevalent among obese patients with cancer and negatively impacts on clinical outcome. Prado et al. ${ }^{6}$ showed that sarcopenic obesity is an independent predictor of survival in obese adult patients with cancer. In the same study, the researchers observed that fat-free mass (FFM) has a poor association with BSA and estimated that variation in FFM among obese patients could result in up to threefold more variation in the effective volume of distribution for chemotherapy administered on the basis of BSA. ${ }^{6}$ This variation suggests that sarcopenic obesity is an unrecognized factor that can contribute to chemotherapy-associated toxic effects in obese patients.

Considering the clinical relevance of sarcopenic obesity, whether sarcopenia or increased adiposity is related to chemotherapy-related toxicity is important to ascertain. Consistent data show that sarcopenia predicts toxicity and complications in patients with cancer, treated with either drug regimens ${ }^{7}$ or surgery, ${ }^{8}$ and also in other diseases. ${ }^{9}$ Thus, body composition analysis should be considered for inclusion in the general assessment of patients with cancer before starting chemotherapy. Many of the available tools for body composition analysis, such as anthropometry, bioimpedance analysis and dual-energy X-ray absorptiometry scans might not be highly accurate or affordable; ${ }^{10}$ however, wholebody CT scans, which many patients with cancer routinely undergo, can be used to easily and precisely measure muscularity. ${ }^{11}$

As correctly noted by Lyman and Sparreboom, ${ }^{1}$ improvements in the tolerability and efficacy of chemotherapy will emerge, hopefully soon, as a more personalized approach to cancer treatment is implemented, taking into account a patient's genetic background and body composition. ${ }^{1}$ The authors also acknowledge that ASCO clinical practice guidelines reflect the best evidence currently available. ${ }^{2}$ Nevertheless, we feel that robust data already exist showing that sarcopenia is a negative prognostic factor for treatment-associated toxicity and, therefore, should be proactively assessed and treated early in all patients with cancer receiving antitumour therapy. This suggestion is in agreement with the provisional clinical opinion issued by ASCO on the early inclusion of palliative care, of which nutrition therapy is central, in the management of patients with cancer. ${ }^{12}$

Department of Clinical Medicine, Sapienza University, Viale del Policlinico 155, 00161 Rome, Italy (A. Laviano, S. Rianda,

F. Rossi Fanelli).

Correspondence to: A. Laviano alessandro.laviano@uniroma1.it

\section{Competing interests}

The authors declare no competing interests.

1. Lyman, G. H. \& Sparreboom, A. Chemotherapy dosing in overweight and obese patients with cancer. Nat. Rev. Clin. Oncol. 10, 451-459 (2013).

2. Griggs, J. J. et al. Appropriate chemotherapy dosing for obese adult patients with cancer: American Society of Clinical Oncology practice guideline. J. Clin. Oncol. 30, 1553-1561 (2012).

3. Dulloo, A. G., Jacquet, J., Solinas, G., Montani, J. P. \& Schutz, Y. Body composition phenotypes in pathways to obesity and metabolic syndrome. Int. J. Obes. (Lond.) 34 (Suppl. 2), S4-S17 (2010).

4. Prado, C. M., Wells, J. C., Smith, S. R., Stephan, B. C. \& Siervo, M. Sarcopenic obesity: a critical appraisal of the current evidence. Clin. Nutr. 31, 583-601 (2012).

5. Laviano, A., Meguid, M. M., Inui, A., Muscaritoli, M. \& Rossi Fanelli, F. Therapy insight: cancer anorexia-cachexia syndromewhen all you can eat is yourself. Nat. Clin. Pract. Oncol. 2, 158-165 (2005).

6. Prado, C. M. et al. Prevalence and clinical implications of sarcopenic obesity in patients with solid tumours of the respiratory and gastrointestinal tracts: a population-based study. Lancet Oncol. 9, 629-635 (2008).

7. Mir, O. et al. Sarcopenia predicts early doselimiting toxicities and pharmacokinetics of sorafenib in patients with hepatocellular carcinoma. PLoS ONE 7, e37563 (2012).

8. Lieffers, J. R., Bathe, O. F., Fassbender, K., Winget, M. \& Baracos, V. E. Sarcopenia is associated with postoperative infction and delayed recovery from colorectal cancer resection surgery. Br. J. Cancer 107, 931-936 (2012). 


\section{CORRESPONDENCE}

9. Montano-Loza, A. J. et al. Muscle wasting is associated with mortality in patients with cirrhosis. Clin. Gastroenterol. Hepatol. 10 , 166-173 (2012).

10. Silver, H. J., Welch, E. B., Avison, M. J. \& Niswender, K. D. Imaging body composition in obesity and weight loss: challenges and opportunities. Diabetes Metab. Syndr. Obes. 3 , 337-347 (2010).

11. Baracos, V. \& Kazemi-Bajestani, S. M. Clinical outcomes related to muscle mass in humans with cancer and metabolic illnesses.
Int. J. Biochem. Cell Biol. 45, 2302-2308 (2013).

12. Smith, T. J. et al. American Society of Clinical Oncology provisional clinical opinion: the integration of palliative care into standard oncology care. J. Clin. Oncol. 30, 880-887 (2012). 